\title{
Description of precision colorimeter
}

This article has been downloaded from IOPscience. Please scroll down to see the full text article.

1987 J. Phys. E: Sci. Instrum. 20882

(http://iopscience.iop.org/0022-3735/20/7/011)

View the table of contents for this issue, or go to the journal homepage for more

Download details:

IP Address: 161.111.22.141

The article was downloaded on 11/12/2012 at 12:00

Please note that terms and conditions apply. 


\section{Description of a precision colorimeter}

\author{
J Campos, A Pons and A Corróns \\ Instituto de Optica, 28006 Madrid, Spain
}

Received 6 May 1986, in final form 3 November 1986

Abstract. This report describes the use of a fully automatic, computer-controlled absolute spectroradiometer as a precision colorimeter. The chromaticity coordinates of several types of light sources have been obtained with this measurement system.

\section{Introduction}

The colour of a light source can be easily determined if the spectral distribution of its emitted light is known.

A fully automatic absolute spectroradiometer was built at the Radiometric Laboratory of the Institute of Optics in Madrid, and an absolute spectroradiometric scale was developed, based on an electrically calibrated pyroelectric radiometer (Carreras and Corrons 1981).

The full system was designed to measure the absolute spectral irradiance of light sources in the visible spectral range, with $2 \%$ estimated uncertainty of measurement. The instrument and standards have been in use since 1980 and after comparison with spectral irradiance standard lamp measurements with the National Bureau of Standards, USA, and participation in the 1985 sixth international intercomparison of measurements of luminous intensity, conducted by CCPR of the Bureau International des Poids et Mesures (the candela has been derived from the mentioned absolute spectroradiometric scale), it is possible to report the actual uncertainty of the system and a stability better than $1.5 \%$ over the last five years.

Since the spectral distribution on the spectroradiometric aperture is given by the emitter radiance, one possible application of this laboratory measurement system is to use it as a colorimeter.

With this instrument, the growing requirements for a reference instrument to perform industrial and scientific colorimetric measurements of light sources in the Instituto de Optica are satisfied.

\section{Measurement method}

The colorimetric problem is to calculate the tristimulus values of the light coming out of any emitter surface. This light provides a colour stimulus at the observer's retina, the colour sensation depending on the spectral distribution of the light.

The tristimulus values $X, Y, Z$ are given by the following expressions:

$$
\begin{aligned}
& X=k \int_{\lambda} S(\lambda) \bar{x}(\lambda) \mathrm{d} \lambda \\
& Y=k \int_{i} S(\lambda) \bar{y}(\lambda) \mathrm{d} \lambda \\
& Z=k \int_{i} S(\lambda) \bar{z}(\lambda) \mathrm{d} \lambda
\end{aligned}
$$

where $k$ is a constant, $S(\lambda)$ is some spectral radiometric magnitude, such as radiant flux, radiance or irradiance, representing the colour stimulus, and $\bar{x}(\lambda), \bar{y}(\lambda)$ and $\bar{z}(\lambda)$ are the spectral tristimulus values.

The tristimulus values may be expressed as dimensionless ratios called 'chromaticity coordinates'. They are related to the tristimulus values simply as:

$$
\begin{aligned}
& x=X /(X+Y+Z) \\
& y=Y /(X+Y+Z) .
\end{aligned}
$$

Therefore, the chromaticity coordinates are uniquely determined when the relative spectral distribution of some radiometric variable is known.

\section{Experimental equipment}

A fully automatic computer-controlled absolute spectroradiometer is used to perform the spectral irradiance measurements.

This instrument consists basically of the following parts (see figure 1): (i) an analyser system, (ii) a detection system and (iii) an automatic data acquisition system.

The analyser system includes a Jarrell-Ash monochromator in a $0.75 \mathrm{~m}$ Czerny-Turner mount. It has a $f / 6.5$ aperture and a maximum resolution of $15 \mathrm{pm}(0.15 \AA)$. It is equipped with adjustable bilateral slits and a wavelength drive scanning system. In front of the monochromator entrance slit we have added a $94 \mathrm{~mm}$ inside diameter sphere, with entrance and exit apertures situated $90^{\circ}$ apart, with the internal surface covered by five coats of a barium sulphate paint.

The integrating sphere is used to provide uniform irradiance over the entrance slit of the optical system, eliminating any undesirable effects that may arise from non-uniformities in the spatial responsivity of the detector or from polarisation effects within the monochromator.

Behind the exit slit of the monochromator, a quartz-window EMI 9558 QB photomultiplier has been installed as the detection system, and the S-20 spectral response covers the wavelength range $250-800 \mathrm{~nm}$. A highly stable, wide-range DC amplifier is used to amplify the photomultiplier signal which, amplified and integrated, is collected by the automatic data acquisition system.

Data acquisition and processing are controlled by the 3054 DL Hewlett Packard automatic data acquisition system. It consists of a HP 85 computer, programmed in BASIC with $32 \mathrm{k}$ central memory, and a HP $3497 \mathrm{~A}$ data acquisition control unit

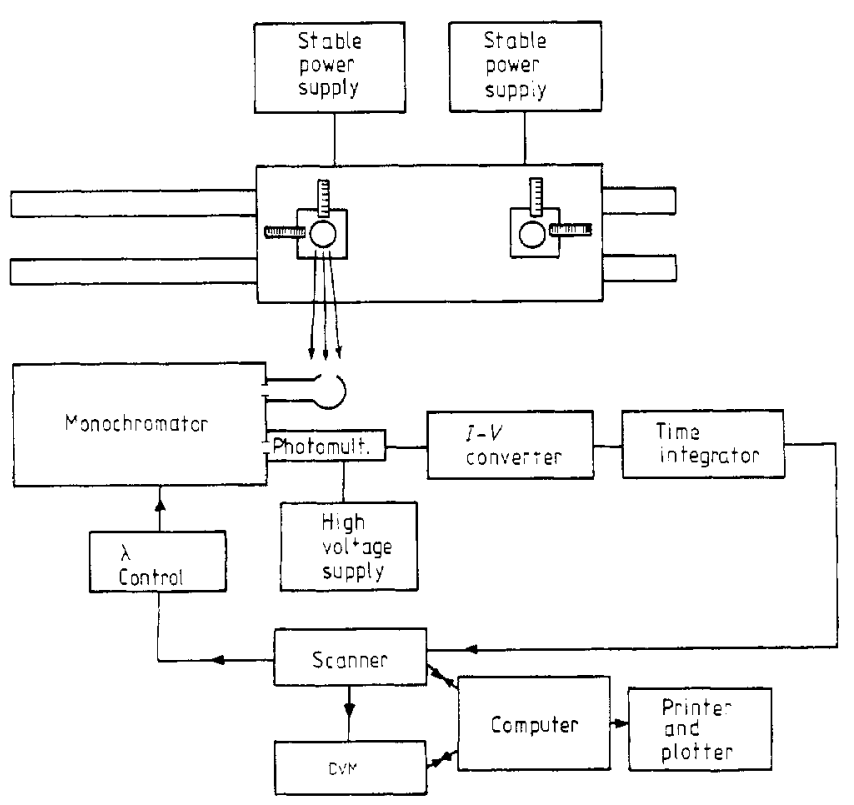

Figure 1. Diagram of the measurement system. 
that includes a digital voltmeter of $10 \mu \mathrm{V}$ resolution, input channels for DC voltage measurements and digital actuator channels.

When the entrance slit of the monochromator is irradiated by a source, the system response will be given by:

$$
r(\hat{\lambda})=k E(\lambda) R(\lambda) \tau(\lambda)
$$

where $\tau(\lambda)$ is the monochromator spectral transmittance, $R(\lambda)$ is the photomultiplier spectral responsivity, $k$ is a constant and $E(\lambda)$ is the source spectral irradiance on the entrance slit. As $R(\lambda)$ and $\tau(\lambda)$ are characteristic functions of the system, we can combine them into another function, $f(\lambda)$, which is the instrument calibration function. Equation (3) then becomes:

$$
r(\lambda)=f(\lambda) E(\lambda)
$$

So if the calibration function is known, the relative spectral irradiance of the source will be immediately determined by measuring the system response, and from equations (1) and (2), the chromaticity coordinates will be also known.

The system also gives the CIELAB coordinates and the psychophysical characteristics of chrome, tone, saturation and luminosity for primary emitters, or clarity for secondary emitters.

Both $1^{\circ}$ and $10^{\circ} \mathrm{CIE}$ spectral tristimulus values are stored so that the coordinates corresponding to either field size can be computed.

The spectroradiometer calibration function was obtained by measuring its spectral response when it was irradiated by the spectral irradiance standard lamp. The lamp is calibrated in absolute values as is described in Carreras and Corróns (1981). This lamp is one of the usual standards in our radiometry laboratory.

The calibration function values obtained were normalised with respect to their maximum and then stored in the computer memory.

\section{Results}

Using the procedure described above, the chromaticity coordinates of the following light sources have been measured.

(i) Real source, designed and developed at the Instituto de Optica (Garcia et al 1980) to reproduce the CIE theoretical illuminant $D_{65}$.

(ii) A combination of an incandescent halide lamp plus red glass filter.

(iii) A combination of the same lamp plus green glass filter.

(iv) A combination of the same lamp plus blue glass filter.

Table 1. Chromaticity coordinates of several illuminants for a

\begin{tabular}{|c|c|c|c|}
\hline Illuminant & $\begin{array}{l}\text { Measured } \\
\text { chromaticity } \\
\text { coordinates }\end{array}$ & $\begin{array}{l}\text { Calculated } \\
\text { chromaticity } \\
\text { coordinates }\end{array}$ & $\begin{array}{l}\text { Colour } \\
\text { differences } \\
\text { CIELAB }\end{array}$ \\
\hline$D_{65}$ & $\begin{array}{l}x=0.3401 \\
y=0.3596\end{array}$ & $\begin{array}{l}x=0.3372 \\
y=0.3581\end{array}$ & $\Delta E=2.5$ \\
\hline $\begin{array}{l}\text { Incandescent } \\
\text { lamp and red } \\
\text { glass filter }\end{array}$ & $\begin{array}{l}x=0.6963 \\
y=0.3036\end{array}$ & $\begin{array}{l}x=0.6962 \\
y=0.3038\end{array}$ & $\Delta E=0.6$ \\
\hline $\begin{array}{l}\text { Incandescent } \\
\text { lamp and green } \\
\text { glass filter }\end{array}$ & $\begin{array}{l}x=0.4265 \\
y=0.4862\end{array}$ & $\begin{array}{l}x=0.4223 \\
y=0.4875\end{array}$ & $\Delta E=3.9$ \\
\hline $\begin{array}{l}\text { Incandescent } \\
\text { lamp and blue } \\
\text { glass filter }\end{array}$ & $\begin{array}{l}x=0.3553 \\
y=0.4210\end{array}$ & $\begin{array}{l}x=0.3530 \\
y=0.4205\end{array}$ & $\Delta E=1.9$ \\
\hline
\end{tabular}
$10^{\circ}$ field of view.
With these light sources the measurement step was $5 \mathrm{~nm}$ from $380 \mathrm{~nm}$ up to $760 \mathrm{~nm}$. The results obtained for the standard observer CIE 1964 (CIE 1971) are summarised in table 1.

To evaluate the accuracy of these results, the relative irradiance of the lamp and the filter transmittance were measured independently and, from them, the chromaticity coordinates were calculated. The results are also presented in table 1 , as well as the colour difference CIELAB.

The spectral transmittance values of the filters were measured with a CARY 17D spectrophotometer. The deviations which can be seen from table 1 are acceptable for measurements done with two different instruments.

The main objective of this work was to obtain a colorimeter to determine precisely the chromaticity coordinates of a light source. Some additional measurements of low radiance sources have been made.

The sources were four light-emitting diodes which are characterised by low radiance and highly spectrally selective outputs. The measurement step with these sources was $1 \mathrm{~nm}$. In table 2 are summarised the average values of the chromaticity coordinates. The uncertainty of the measurements is indicated by the standard deviation.

Table 2. Chromaticity coordinates for light emitting diodes for a $10^{\circ}$ field of view.

\begin{tabular}{lll}
\hline LED & $\begin{array}{l}\text { Chromaticity } \\
\text { coordinates }\end{array}$ & $\begin{array}{l}\text { Standard } \\
\text { deviation }\end{array}$ \\
\hline Green I & $x=0.2341$ & $\sigma=0.0005$ \\
Green II & $y=0.4200$ & $\sigma=0.0005$ \\
& $x=0.4663$ & $\sigma=0.0005$ \\
Red I & $y=0.5321$ & $\sigma=0.0001$ \\
Red II & $x=0.6974$ & $\sigma=0.0001$ \\
& $y=0.3026$ & $\sigma=0.0006$ \\
& $x=0.6971$ & $\sigma=0.0021$ \\
& $y=0.2974$ & $\sigma=0.0005$ \\
\hline
\end{tabular}

\section{Conclusions}

Automatic computer-controlled spectroradiometers can be used as precision colorimeters. With the adaptation of the instrument described, the chromaticity coordinates of several light sources (a real source that reproduces the theoretical illuminant $D_{65}$ and a combination of incandescent lamp plus coloured glass filters) have been obtained.

Chromaticity coordinates of low radiance and highly spectrally selective light sources (LEDS) can be also measured.

The described instrument has been used to perform spectroradiometric and colorimetric measurements of light sources in the Instituto de Optica of Madrid.

\section{References and further reading}

Carreras C and Corróns A 1981 Absolute spectroradiometric and photometric scales based on an electrically calibrated pyroelectric radiometer

Appl. Opt. 201174

Christie J S and McConnell G 1977 A new flexible spectrophotometer for color measurement Proc. of Color 77 (Bristol: Adam Hilger) p 309

CIE 1971 Colorimetry Publication no 15

García A, Corróns A and Pons A 1980 Realización de una fuente patrón $D_{6 s}$ para colorimetria Opt. Pur. Apl. 13103 
Hengstberger F and Appenroth T 1983 The development of a fully automated Absolute Radiometer CSIR (Pretoria) Research Report 331

Wright W D 1977 The spectrophotometer as a tool in art technology

Proc. of Color 77 (Bristol: Adam Hilger) p 310

\section{A method for analysing elliptically polarised light over a range of wavelengths}

M C K Wiltshire and M R Lewis

GEC Research Ltd, Hirst Research Centre, East Lane, Wembley, Middlesex HA9 7PP, UK

Received 23 October 1986, in final form 12 December 1986

\section{Introduction}

Elliptically polarised light can be generated in many ways. One common method is by passing linearly polarised light through a birefringent plate. Depending on the orientation of the plane of polarisation of the incident light with respect to the plate's principal axes, and on the retardation of the plate, the emergent light may be linearly, circularly or elliptically polarised. If the light is polychromatic, then the state of polarisation of the emergent light is generally a function of wavelength. Another situation in which elliptically polarised light is often generated is in the study of the optical properties of chiral liquid crystals. These materials are both optically active and birefringent, and both these properties are wavelength dependent. An initially linearly polarised light beam is, in general, rendered elliptical on passage through these liquid crystals, and the azimuth and ellipticity of the light are again functions of wavelength.

Conversely, the measurement of the azimuth and ellipticity as a function of wavelength of the emergent light permits a sample such as those mentioned above to be optically characterised. Another area where wavelength-dependent effects are important is in the study of polymers, where optical rotatory dispersion is a valuable measurement.

The conventional method for analysing elliptically polarised light requires the light to be monochromatic. Adjustment of an analyser and an appropriate quarter-wave plate enables the azimuth and ellipticity to be determined. This technique can be automated by using suitable compensators driven by the output of a phase-sensitive detection system. Highly accurate results can be achieved, but the process is inherently slow and not well suited to wavelength scanning. The advent of sensitive optical multichannel detectors (e.g. Si diode arrays) makes it both desirable and possible to develop a method to determine the azimuth and ellipticity of elliptically polarised light without requiring the light to be monochromatic. In this paper, we describe such a technique, by which these properties may be obtained as a function of wavelength across the full wavelength window of the detector system, without the need for wavelength scanning. 